COMPUTER SCIENCE

\title{
IMPROVEMENT OF ANTHROPOGENIC ENVIRONMENTAL LOADS ASSESSMENT METHODS USING MODERN INFORMATION TECHNOLOGIES
}

\author{
Bakunova Oksana Mikhailovna, Master of Technical Sciences, Senior Lecturer, Belarusian State \\ University of Informatics and Radio Electronics Minsk, Republic of Belarus \\ Abraztsova Volha Mikalaeuna, Ph.D., Belarusian State University of Informatics and Radio \\ Electronics Minsk, Republic of Belarus \\ Bakunov Alexander Mikhailovich, Master of Technical Sciences, Senior Lecturer, Belarusian State \\ University of Informatics and Radio Electronics Minsk, Republic of Belarus \\ Burkin Anton Vladimirovich, software engineer, BRIMIT company, Minsk, Belarus
}

DOI: https://doi.org/10.31435/rsglobal_wos/31102019/6737

\section{ARTICLE INFO}

Received: 18 August 2019

Accepted: 14 October 2019

Published: 31 October 2019

\section{KEYWORDS}

innovations, developing

technologies, the modern platforms

of programming, ecology.

\begin{abstract}
The innovative path of human development is characterized by the active use of information technology in any field. This is due to the rapid development of high technologies and, accordingly, with the advent of new data analysis tools. One of the rapidly developing areas of application of information technology, to which there is great interest in society, the state, commercial organizations, is high-quality forecasting.
\end{abstract}

Citation: Bakunova Oksana Mikhailovna, Abraztsova Volha Mikalaeuna, Bakunov Alexander Mikhailovich, Burkin Anton Vladimirovich. (2019) Improvement of Anthropogenic Environmental Loads Assessment Methods Using Modern Information Technologies. International Academy Journal Web of Scholar. 10(40), doi: 10.31435/rsglobal_wos/31102019/6737

Copyright: (C) 2019 Bakunova Oksana Mikhailovna, Abraztsova Volha Mikalaeuna, Bakunov Alexander Mikhailovich, Burkin Anton Vladimirovich. This is an open-access article distributed under the terms of the Creative Commons Attribution License (CC BY). The use, distribution or reproduction in other forums is permitted, provided the original author(s) or licensor are credited and that the original publication in this journal is cited, in accordance with accepted academic practice. No use, distribution or reproduction is permitted which does not comply with these terms.

Big Data technologies have been successfully applied in this area. At the same time, important directions of the sustainable development strategy are goals directly or indirectly related to the state of the environment - such as ensuring the availability and rational use of water resources, the sustainability of cities and towns, including improving public transport, creating green public places and improving urban planning and improvement, combating climate change, preserving marine systems and terrestrial ecosystems, developing sustainable patterns of consumption and production, including their efficient allocation of shared natural resources as well as a reasonable accommodation of toxic waste and contaminants, stimulating and motivating industries, businesses and consumers to recycle and reduce the amount of waste [7]. This work is a synthesis of these two areas - the use of modern information technologies for processing geoecological information and building a system for predicting the level of comfort of living in the territorial units of the Republic of Belarus.

To ensure sustainable development of the Republic of Belarus, comprehensive measures should be provided to protect the atmosphere, protect and rational use of land, forest, mineral resources, water resources, to promote sustainable agriculture and rural development, to preserve biological diversity, and the environmentally safe use of biotechnologies and toxic chemicals for the environmentally sound disposal of hazardous, solid and radioactive waste. 
The sustainable development strategy should consider environmental protection and rational use of natural resources not as an end in itself, not in isolation from the development process, but as an integral part of it. In general, nature management should be carried out using such methods and on such a scale that they can achieve the necessary economic development under the indispensable condition of ensuring equal opportunities and preserving the environment for future generations. This requires the greening of the economy, the transition to a new stage of material culture, compatible and balanced with natural resource potential. The Sustainable Development Strategy seeks to harmonize social, economic and environmental development as equivalent complementary components in a single balanced complex "man - environment - economy" [7]. Therefore, the urgent problem is the development of a method for processing heterogeneous environmental indicators, bringing them to a single presentation form, the development of an indicator linking the above components of the "man environment - economy" system and the creation of monitoring and forecasting systems for the state of the environment. Such an indicator in this work is the level of comfort of living (UKP).

Currently, in connection with the growth of industry, emissions, the relevance of anthropogenic pressure assessment is increasing. Factors of anthropogenic impact on the environment and environmental risk are closely related, with an increase in anthropogenic impact, the likelihood of an event having adverse effects on the environment - environmental risk - also increases. Data on levels of harmful and dangerous factors are important both for assessing the level of environmental pollution and for assessing the risk of morbidity and mortality.

In real conditions, anthropogenic influence on the territory of the administrative districts may be divided into several components, main of which are: industrial, agricultural, transport and demographic load. Equally important is the level of physical contamination, including noise, vibration and ionizing radiation.

In the assessment of anthropogenic impact and of calculating the level of PPD, it is important to reasonably select the primary units for research, characterized by a sufficient homogeneity of the economic use and environmental conditions.

At the present stage of development of society, protection of environment is paid much attention. This is due to increasing anthropogenic pressure due to the establishment of new industries and the need to limit the adverse effects of pollutants on public health.

One way of monitoring the state of the environment are stationary posts of observation. Monitoring atmospheric air is carried out in 19 cities and industrial centers. Regular observations covered the territory, which is home to $87 \%$ of the population of big and medium cities in the country.

Currently, human economic activity has a significant impact on the biosphere, that is why environmental issues, environmental protection, assessing the impact of environmental factors on human health, public availability of environmental information are important. The software allows you to perform a comprehensive assessment of anthropogenic load in the administrative area, including the consideration of pollution in different environments, types of pollutants, specific pollutants.

The software package produces statistical processing of data received from fixed and mobile monitoring stations for the environment. figures 1 and 2 present data on the following features:

- environment;

- type of pollutant;

- pollutant;

- the city;

- type of observation post;

- observation post.

The database contains the values of maximum permissible concentrations that correspond to the currently established regulatory legal acts the user can see.

The user can set the search parameters that will be reflected in the report, the report can be generated for a specific administrative territory, a specific pollutant, polluted environment and a period of time.

The reports (Figs. 3 and 4) have two possibilities of visualization according to private indicators: construction of linear diagrams against the background of values averaged over all administrative territories or in the form of a report generated in .pdf format with color highlighting of permissible / unacceptable concentrations. The complex indicator is visualized by filling in the administrative territories with a certain color, depending on the value of the complex indicator. 


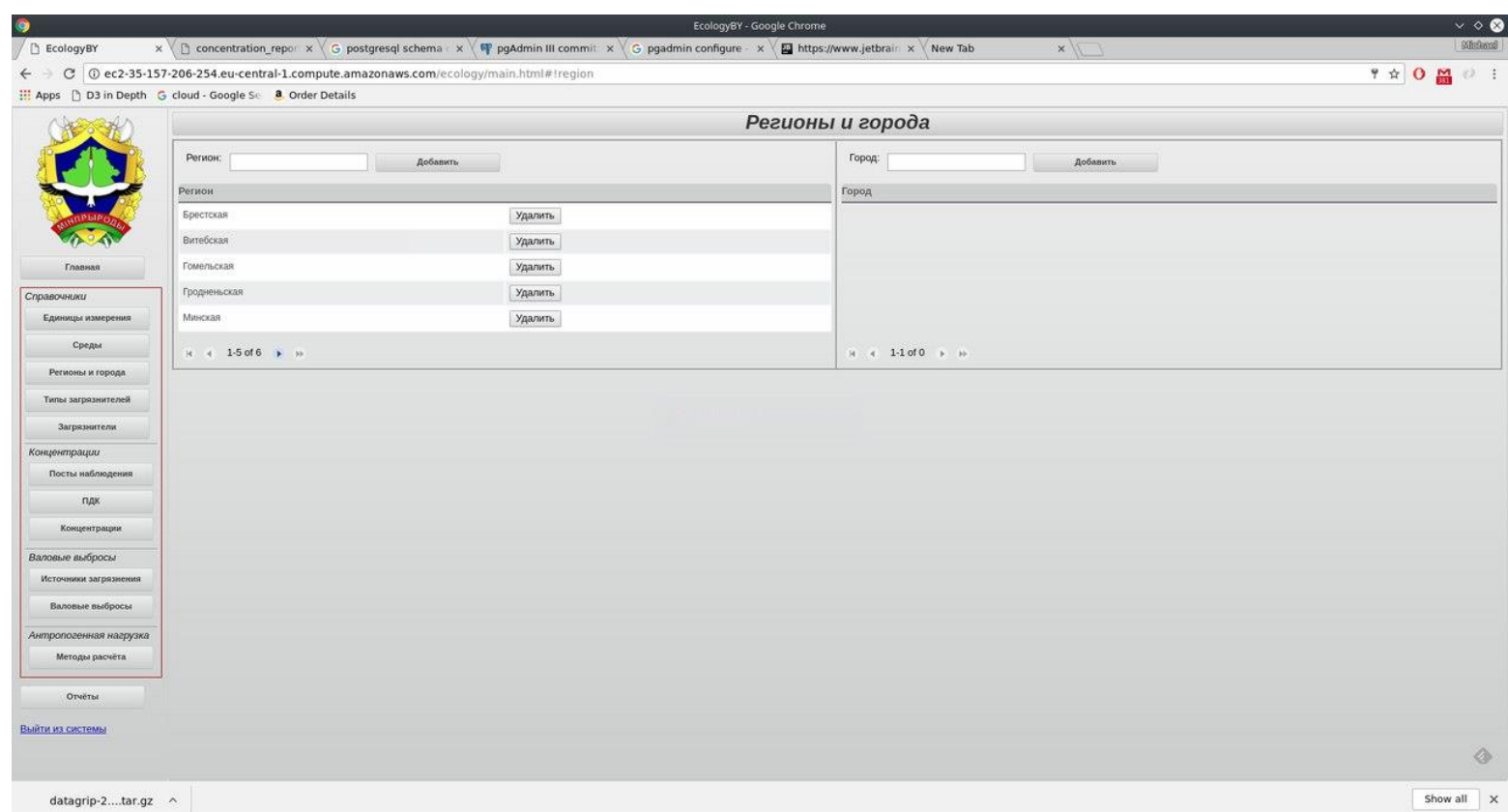

Fig. 1 Data on cities

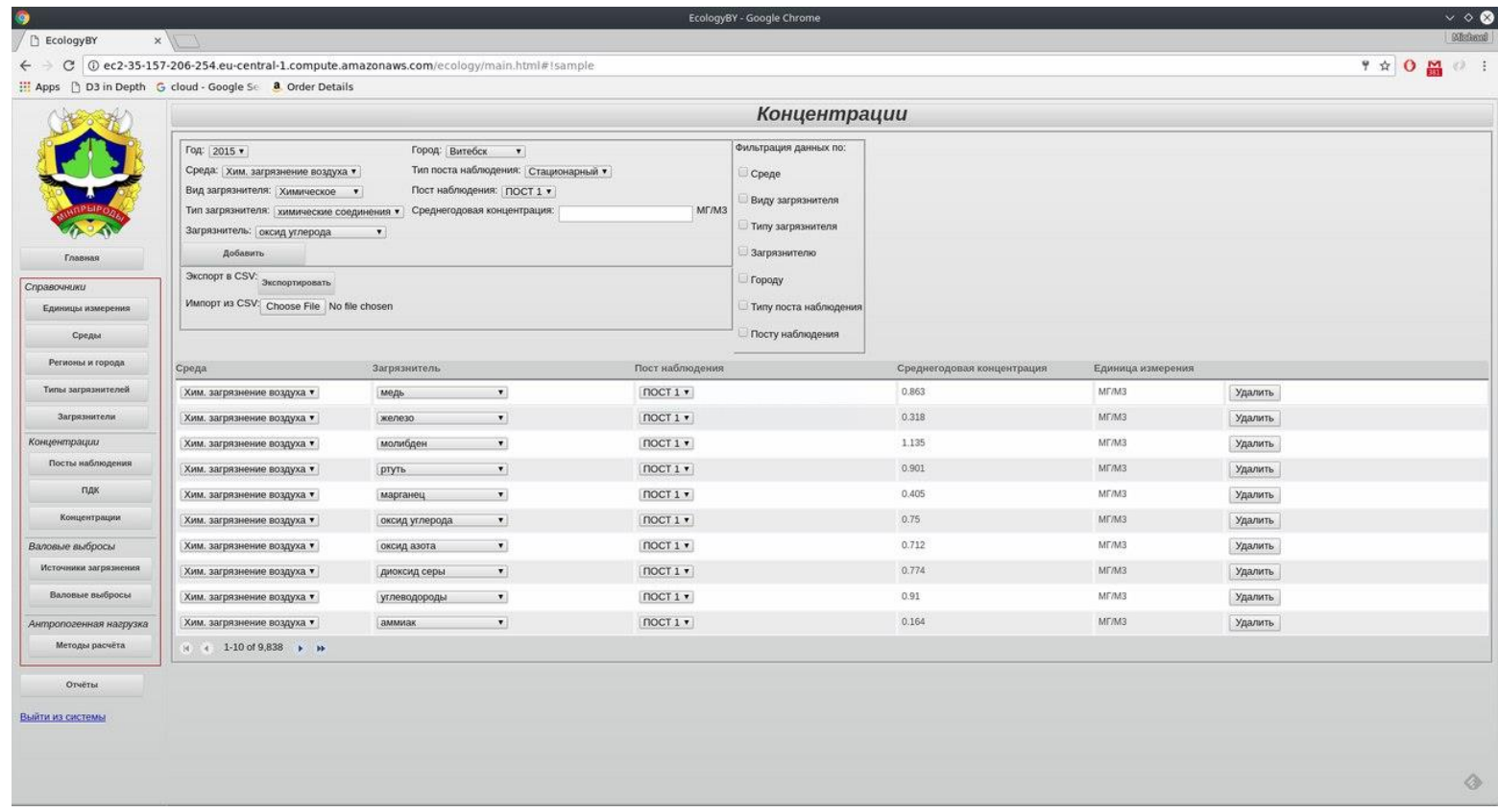

Fig. 2 Data on pollutant concentrations

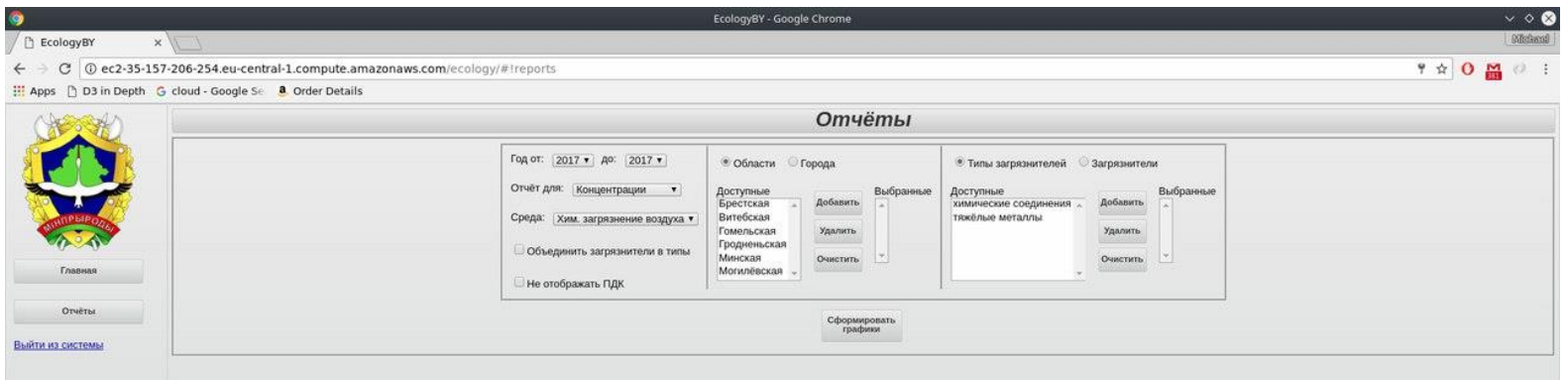

Fig. 3. Setting report parameters. 


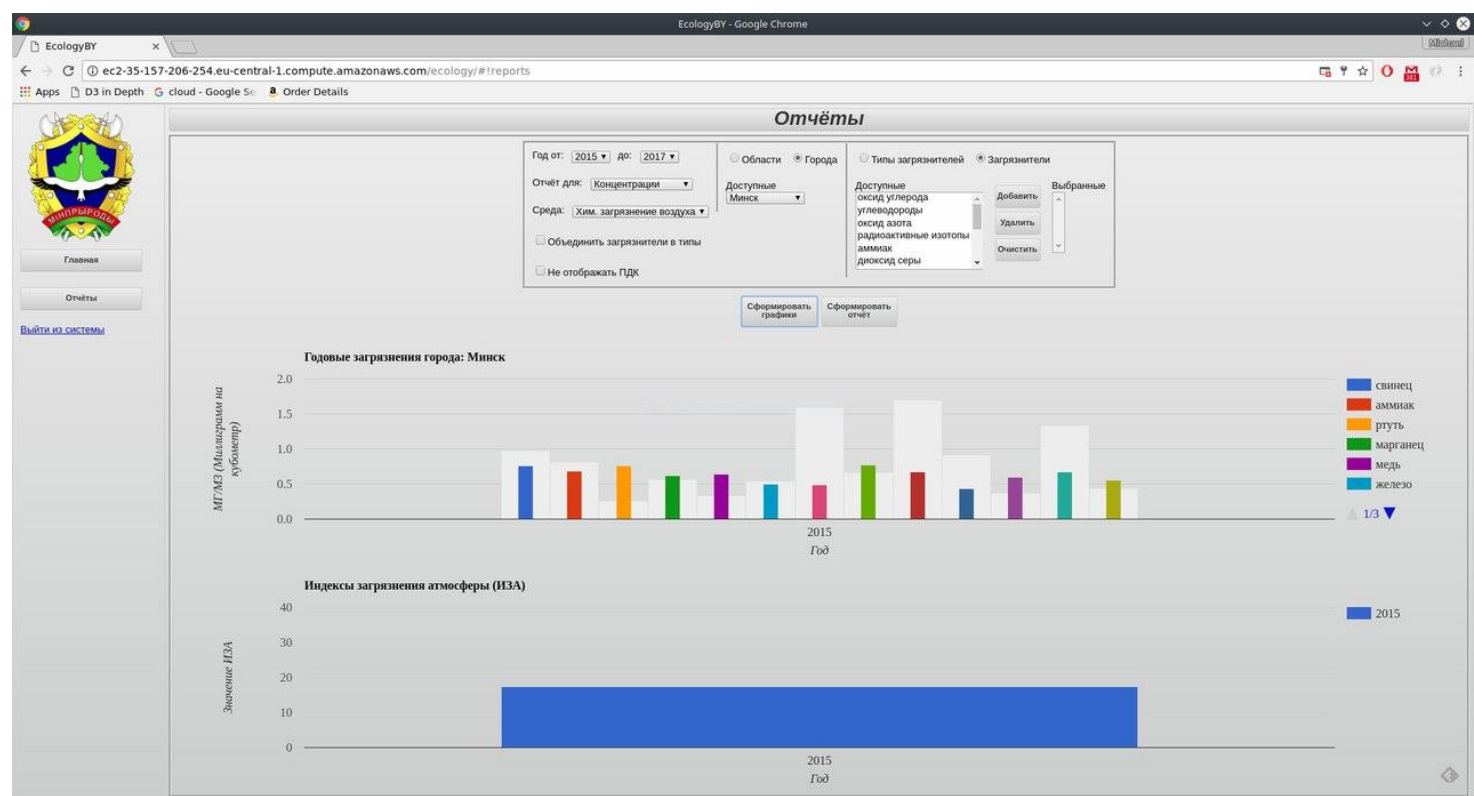

Fig. 4. Visualization of the results in the form of linear diagrams according to the data for the city of Minsk

To calculate the PPD is based on a mathematical model with regression equations that will allow us to more adequately consider the extent of the influence of anthropogenic impacts on territorial unit.

Assessment of the level of comfort of living in a certain area of the city is a priority in the research of modern ecology. For its calculation, and storage of information requires a large amount of data.

Assessment of the level of comfort of living were cited in three areas of the environment - air, soil cover and water resources.

The task of a regression method neural network modelling to solve different types of problems: multi-layer perceptron, linear networks, radial basis function and generalized regression network. The linear model does not differ from ordinary linear regression, but in the language of neural networks represented by the network without intermediate layers, in which the output layer contains only linear elements (i.e. elements with linear activation function). It is possible to train the linear network using the standard algorithm for linear optimization.

In general, the problem of forecasting time series with Ann is reduced to the following sequence of steps:

The problem of forecasting of environmental situation in the territorial unit of the Republic of Belarus is reduced to the following stages: collecting data for raining (the stage considered one of the most complicated ones);

- preparation and normalization of data (reduction to BP type);

- choice of ANN topology (at this stage, a decision is made on the number of layers and the presence of feedback);

- empirical (by experiment) selection of ANN characteristics;

- empirical selection of learning parameters;

- ANN training;

- verification of training on the adequacy of the task;

- adjustment of parameters taking into account the previous step, final training;

- verbalization of ANN (minimized description using several algebraic or logical functions) for the purpose of further use.

- analysis and preparation of a sample of experimental data for their computer processing.

For forecasting, we used the data of annual reports of the National Environmental Monitoring System of the Republic of Belarus for the period from 2006 to 2018.

Conclusions. When developing monitoring methods and systems, many parameters must be taken into account. For this period of time, there are only software tools and methods that take measurements of pollution indicators for individual components of the environment. Evaluation of individual components is important, but it does not allow evaluating in a complex; therefore, a method 
that generally considers all anthropogenic loads is of scientific interest. Thanks to the integrated data processing, this method has significant advantages and a wider range of applications.

The undoubted pluses include the fact that all disparate indicators that are difficult for control and understanding by non-specialists are combined into one, which greatly simplifies the use of calculation results and provides a backlog in the future for the creation of an information resource on which the ecological state will be displayed in real time .

The minus of the information and averaging of the assessment should not be neglected, namely that due to the possibility of fluctuations of various indicators, a comprehensive assessment may remain within the norm, and some of the indicators will be above the norm. To eliminate this drawback, it is necessary, when designing a software tool, to introduce additional control modules, but this will allow it to be developed in the future to a full-fledged expert system.

\section{REFERENCES}

1. Environmental Indicators and Indicators-based Assessment Reports - Eastern Europe, Caucasus and Central Asia // ECE/CEP/140

2. UNECE Convention on Access to Information, Public Participation in Decision-making and Access to Justice in Environmental Matters (Aarhus Convention) [Electronic resourse]. URL: https://www.unece.org/env/pp/treatytext.html (access date: 23.10.2017)

3. Hygienic standards 2.1.7.12-1-2004 "The list of maximum permissible concentrations (MPC) and tentatively permissible concentrations (MPC) of chemicals in the soil".

4. The National Environmental Monitoring System of the Republic of Belarus: Observation Results, 2015 / Edited by M.A. Eresko [Electronic resource]. Electron. text, graph. data. (55.5 Mb), - Minsk, "Bel SIC" Ecology ". - 2016. - 1 electron. opt. disc (CD-ROM): color; $12 \mathrm{~cm}$. - System. requirements: Pentium II and higher; Windows XP

5. Bakunova O. M., Kalitenya I. L., Bakunov A. M., Paluiko A. F., Antonov E. D., Grechko I. S. Use of neural networks in education. INTERNATIONAL ACADEMY JOURNAL Web of Scholar 1 (19), Vol. 1, - Warsaw, Poland, 2018 S. 8 - 11

6. Bakunova O. M., Khmelevskaya A. L., Belikov A. S., Mironchik A. N., Agapkin L. M., Chuchval A.Yu. Using modern approaches and neural networks for quality education in universities // I International Symposium "Humanities and Social Sciences in Europe: Achievements and Prospects" - Vienna, Austria 2018

7. National Strategy for Sustainable Socio-Economic Development of the Republic of Belarus for the Period Until 2020 / National Commission on Sustainable Development Rep. Belarus; Editorial Board: Ya.M. Alexandrovich et al. - Mn.: Unipak. - $200 \mathrm{~s}$ 